\title{
Aero-thermal simulations of the TMT Laser Guide Star Facility
}

Konstantinos Vogiatzis, Corinne Boyer, Kai Wei, Jinlong Tang, Brent Ellerbroek

Konstantinos Vogiatzis, Corinne Boyer, Kai Wei, Jinlong Tang, Brent Ellerbroek, "Aero-thermal simulations of the TMT Laser Guide Star Facility," Proc. SPIE 9148, Adaptive Optics Systems IV, 914868 (7 August 2014); doi: $10.1117 / 12.2057208$

Event: SPIE Astronomical Telescopes + Instrumentation, 2014, Montréal, Quebec, Canada 


\title{
Aero-thermal simulations of the TMT Laser Guide Star Facility
}

\author{
Konstantinos Vogiatzis ${ }^{1}$, Corinne Boyer ${ }^{1}$, Kai Wei $^{2}$, Jinlong Tang $^{2}$, Brent Ellerbroek ${ }^{1}$ \\ ${ }^{1}$ TMT Observatory Corporation, Pasadena, CA 91105, USA \\ ${ }^{2}$ Institute of Optics and Electronics, Chengdu, China
}

\begin{abstract}
The Laser Guide Star Facility (LGSF) system of the Thirty Meter Telescope (TMT) will generate the artificial laser guide stars required by the TMT Adaptive Optics (AO) systems. The LGSF uses multiple sodium lasers to generate and project several asterisms from a laser launch telescope located behind the TMT secondary mirror. The laser beams are transported from a location below the primary mirror to the launch telescope using conventional optics to relay the beams along the telescope structure. The beams and relay optics are enclosed into hermetic ducts for safety reasons and to protect the optics against the environment.

A Computational Solid Fluid Dynamics (CSFD) model of the LGSF ducts has been developed. It resolves the duct thickness, laser beam transfer lenses, mirrors and their framework for most of the laser beam path that is subject to significant temperature gradients and/or large vertical change. It also resolves the air inside the duct and its thermal interaction with the aforementioned components through conjugate heat transfer. The thermal interaction of the laser beam with the optics is also captured. The model provides guidance to the LGSF design team and a first estimate of the laser beam stability performance and requirement compliance.

As the telescope structure design has evolved in the recent years, a new optical path has been proposed for the LGSF. Both the original and the new optical paths are compared against optical, mechanical and other telescope performance related criteria. The optical performance criteria include a first order analysis of the optical turbulence generated within the ducts. In this study simulations of the thermal environment within the ducts of the two candidate paths are performed and conclusions are drawn.
\end{abstract}

Keywords: Computational Solid-Fluid Dynamics, Laser Guide Star Systems, Ground Based Telescopes

\section{INTRODUCTION}

\subsection{Overview}

The Laser Guide Star Facility (LGSF) is one of the TMT main Adaptive Optics (AO) systems [1],[2] It is being designed by the Institute of Optics and Electronics (IOE) in Chengdu China. It is responsible for generating the artificial laser guide stars required by the TMT Laser Guide Star (LGS) AO systems. The LGSF uses multiple sodium lasers to generate and project up to 4 different LGS asterisms with up to 9 guide stars from a laser launch telescope (LLT) located behind the TMT secondary mirror. The LGSF consists of 4 sub-systems as illustrated in Figure 1:

- The Laser System, attached to the side of the $-\mathrm{X}_{\mathrm{ECRS}}$ elevation journal (side closest to the primary mirror). The Laser System includes up to 9 Laser Units. Each Laser Unit (LU) consists of one Laser Head (LH) and one Laser Electronics (LE). The Laser Heads are attached to the inside of the $-\mathrm{X}_{\mathrm{ECRS}}$ Elevation Journal and the Laser Electronics are mounted on the Laser Platform directly facing the Laser Heads as shown in Figure 2. The laser beams are transported from LH1 to LH9 then up via the optical path to the LGSF Top End and then projected onto the sky from the Laser Launch Telescope.

- The Beam Transfer Optics (BTO) Optical Path (OP), which is responsible for transporting the laser beams from the output of the Laser System up to the LGSF Top End. It uses a $3 \times 3$ square pattern and a relay of conventional fold mirrors. The laser beams are fully enclosed within hermetic ducts for laser safety protection and also to protect the optics for the telescope dusty environment. The Optical Path consists of two main sections:

- The laser platform section located on the laser platform, which consists of the laser benches (one per laser head) and the sections of horizontal duct located between each laser benches.

- The elevation section, which transports the laser beams from the end of the laser platform section to the LGSF Top End. Two paths are being considered for the elevation section and TMT (referred in this paper as the baseline OP and alternative OP) and IOE are in the process of selecting the best optical path for the LGSF as we are writing this paper. 
- The LGSF Top End, which consists of the LGSF components located behind the TMT secondary mirror (Figure 3). The role of the LGSF Top End is mainly to format the beams into the desired asterism (using the Asterism Generator) and to launch the asterism onto the sky via the Laser Launch Telescope Assembly. It also includes the diagnostics systems to align and calibrate the LGSF system (i.e. Diagnostic Bench sub-system and Acquisition sub-system).

- The laser safety system, which consists of three main sub-systems: i) an aircraft detection system, ii) a laser traffic control system dedicated to the prevention of beam collisions with neighboring telescopes, and iii) a laser interlock system dedicated to shuttering the laser beams in response to events received form the aircraft detection system, the laser traffic control system or any faults or emergency from the LGSF or AO systems or telescope.

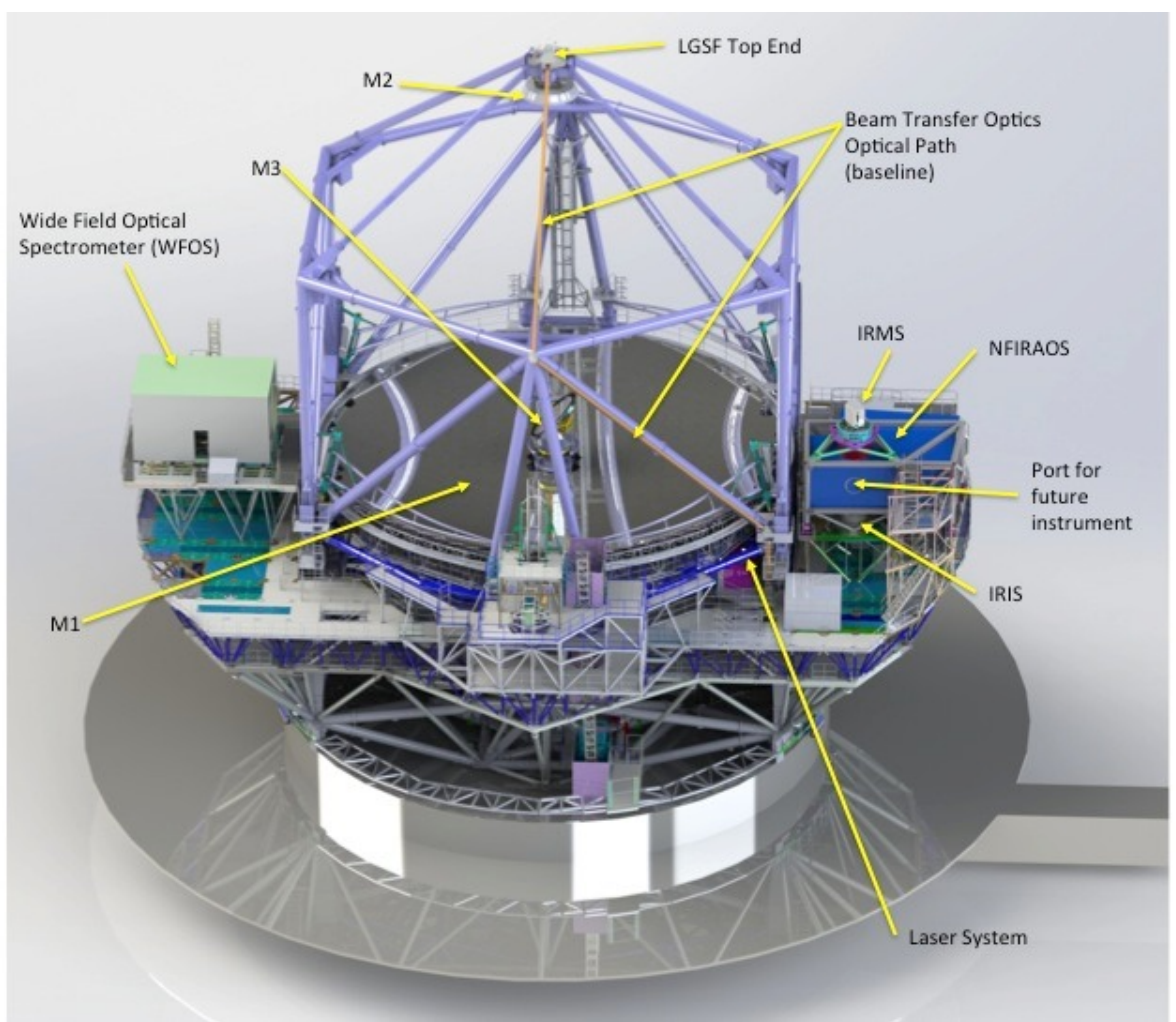

Figure 1. TMT telescope with first light instrumentation and AO systems (LGSF and NFIRAOS) viewed from the back of the telescope

\subsection{Optical Path trade study}

As the telescope structure design has evolved in the recent years, a new optical path has been proposed for the LGSF. This path was not available with the previous telescope structure design with only three secondary mirror support struts. The two candidate paths include a total of only four folds to transport the laser beams from the output of the laser platform to the entrance of the LGSF Top End. The two paths are described in Figure 4. The two paths are compared against the following criteria:

- Optical performance, including i) the optical design and layout and in particular the number of folds, ii) the angle of incidence of the different folds, which affects the overall optical path throughput, iii) a first order gravitational flexure and beam propagation and compensation analysis, and iv) a first order analysis of the thermal turbulence within the ducts (This is the subject of this paper).

- Mechanical performance, including i) the layout of the mechanical design and the distance between the ducts and the telescope structure, ii) the weight of the optical path, iii) the impact on the LGSF Top End design, iv) the access to the different components of the LGSF optical path, and v) the interference with the other telescope systems.

- Other telescope performance, including the wind jitter analysis. 


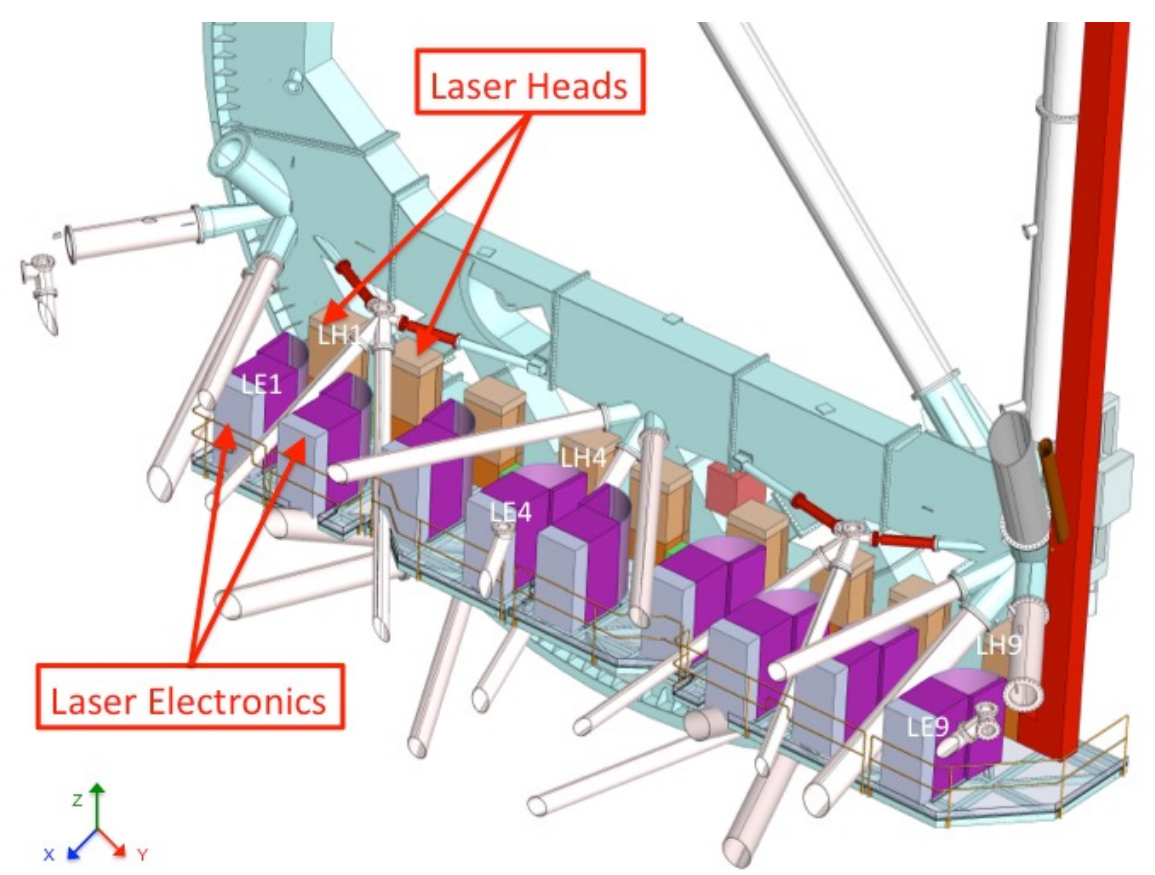

Figure 2. TMT Laser System including up to 9 laser units. Each laser unit is composed of one laser head (LH - light brown) attached to the elevation journal and one laser electronics (LE - purple) mounted on the laser platform. The laser beams are formatted into a square pattern at the output of the laser platform and sent up to the elevation structure via the elevation section of the optical path (red duct).

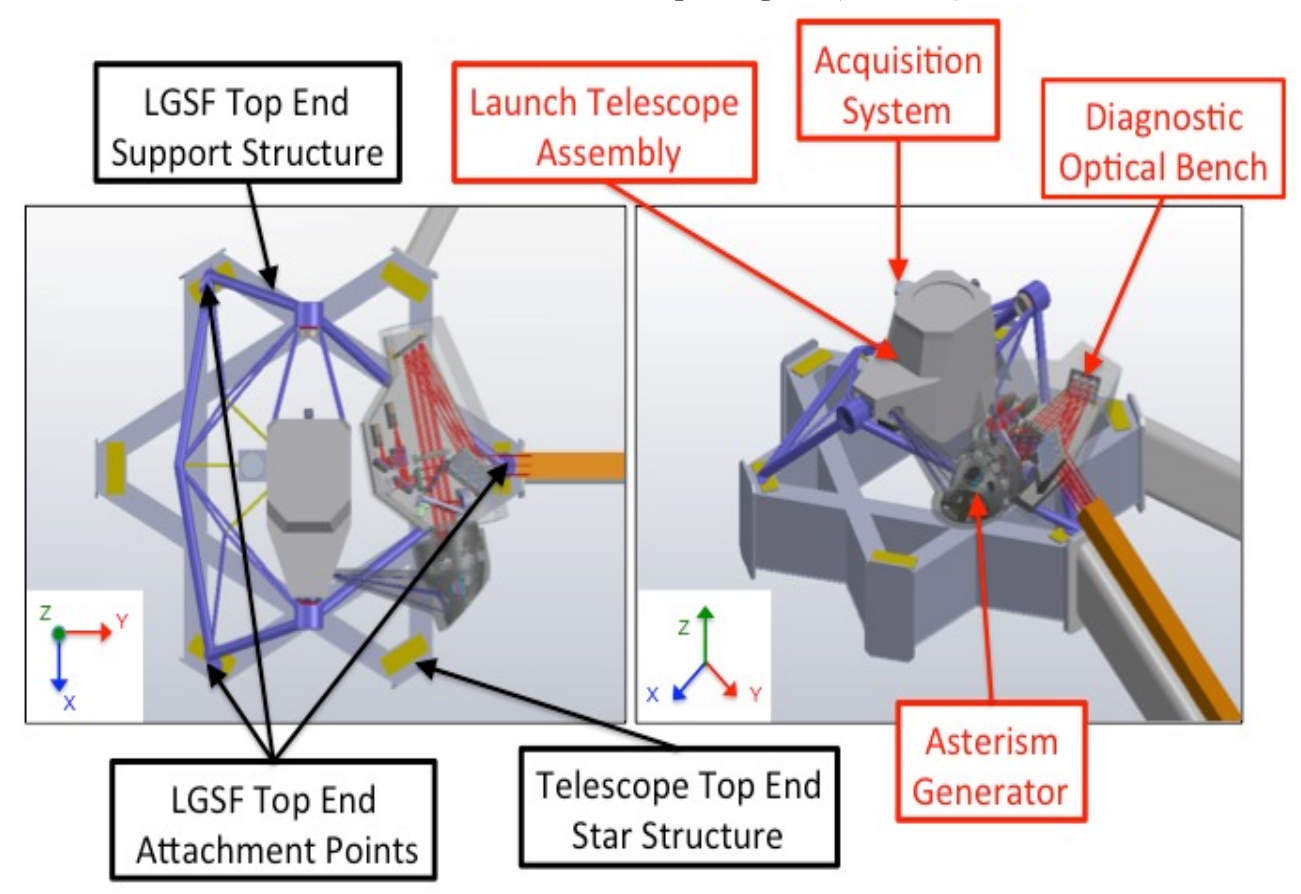

Figure 3. TMT LGSF Top End (baseline OP), including the Asterism Generator, the Launch Telescope Assembly and the diagnostic systems for LGSF alignment and calibration (Optical Bench and Acquisition sub-systems) 


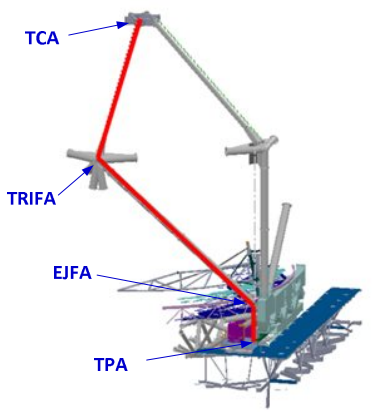

LGSF Baseline Optical Path

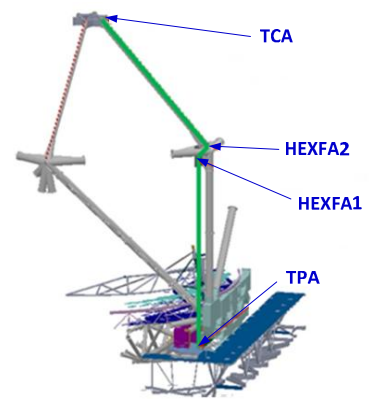

LGSF Alternative Optical Path

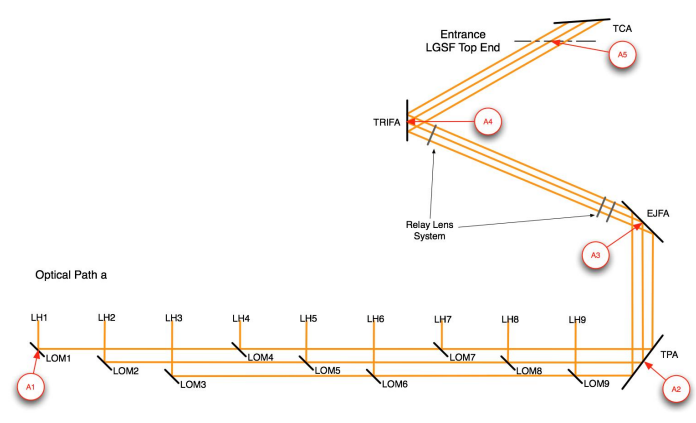

LGSF Baseline Optical Path Layout

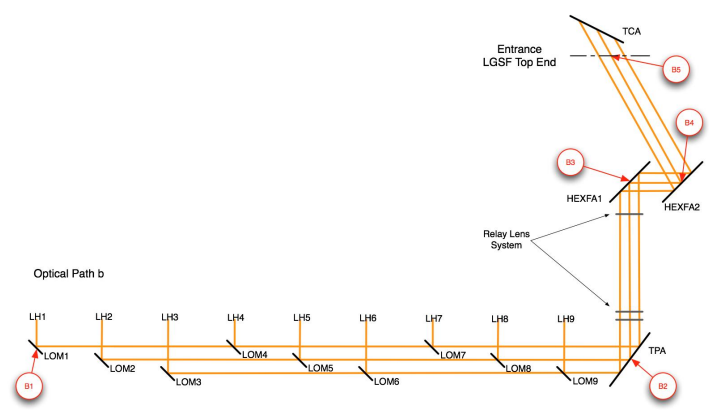

LGSF Alternative Optical Path Layout

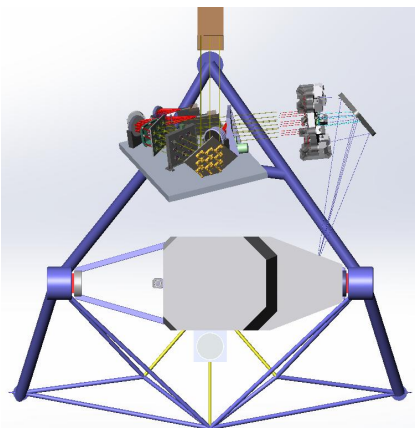

LGSF Top End Layout for Baseline OP

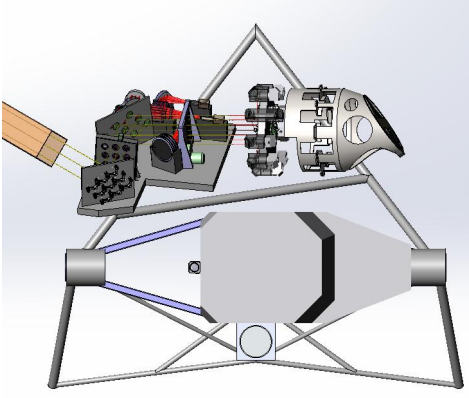

LGSF Top End Layout for Alternative OP

Figure 4. LGSF optical path options. Baseline optical path (red) has one large angle fold (EJFA). The alternative optical path (green) has no large angle folds, but requires additional structure to attach the vertical section of the optical path to the telescope.

\section{AERO-THERMAL MODELING}

\subsection{Overall description}

The model presented herein is a conjugate heat transfer aero-thermal model, that solves for the velocities of the air inside the ducts as well as the temperature of both the air and the solid components. It is a multi-region model similar in principle to the TMT Telescope Structure model described in [3] and the TMT Observatory CFD model described in [4]. Each region represents a fluid volume or a solid component that are connected through air-to-solid or solid-to-solid interfaces. The grid of each region has been generated in such a way that each interface facet is common to the two cells of the two regions involved. Convection, conduction and radiation heat transfer between regions is taking place through all interfaces.

\subsection{Model geometry}

The exterior of the CAD models used can be seen in Figure 5. In the reference coordinate system the Elevation Axis is along $\mathrm{X}$ and the Optical axis is along $\mathrm{Z}$. The baseline model includes $47.3 \mathrm{~m}$ of path-length, incorporating TPA at the Elevation Journal platform level, EJFA a few meters above that and TRIFA at the hex ring level. The alternative model includes $43.2 \mathrm{~m}$ of path-length, incorporating TPA at the Elevation Journal platform level, as well as HEXFA1 and HEXFA2 at the hex ring level.

The resulting CSFD model consists of five distinct regions:

- "Duct", including enclosures

- Interior duct "Air"

- $\quad$ "Lenses", 3 sets of 9

- $\quad$ "Mirrors", 3 sets of 9

- $\quad$ Support "Frames" for the optics, 6 

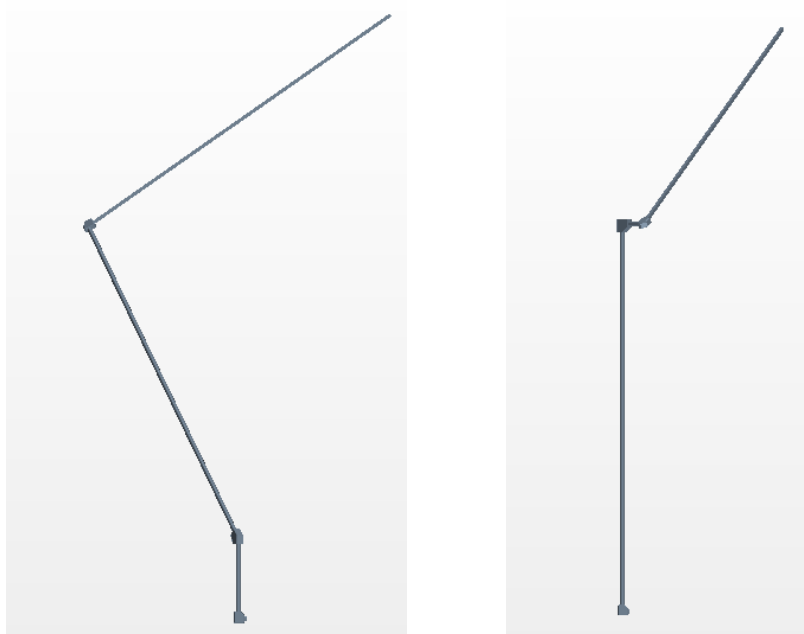

Figure 5. LGSF BTO CSFD CAD optical path models for the baseline OP (left) and the alternative OP (right)

\subsection{Environment}

The model uses environmental inputs as formulated in the TMT Monte Carlo framework [5], along with results generated by the TMT Aero-thermal Modeling Framework [6], using the updated TMT Heat Dissipation Budget [7]. More specifically, the expected ambient temperature night-time temporal behavior is a result of statistically averaging the temperature records of the Monte Carlo framework. The temperature day-time temporal and spatial behavior was taken from CFD simulations for the Air Handling Unit performance. The enclosure surface temperatures are estimated by an FEA model that uses the entire Monte Carlo framework environmental and telescope orientation record. Finally, convective heat transfer coefficients are provided by the matrix of TMT CFD simulations under various wind speeds and telescope orientations.

After studying the latest expected inputs and estimated environment, we made the following selections for the environmental conditions, as also reported in [3].

Input ambient temperature $\mathrm{TA}(\mathrm{t}, \mathrm{Y})(\mathrm{t}$ is time in hours, $0-24, \mathrm{Y}$ in $\mathrm{m}$ in the reference system direction):
Night:
$277.6-\mathrm{t}^{*}\left(\mathrm{e}^{-\mathrm{t}}+0.3\right) \mathrm{K}$
Day (enclosure interior): $\left[274.7+0.8 *\left(1-\mathrm{e}^{-(\mathrm{t}-12) / 1.5)}\right]+0.06 * \mathrm{Y} \mathrm{K}\right.$
(Eq. 1)
(Eq. 2)

Note also that the telescope is pointing at horizon after sunrise (hence the $\mathrm{Y}$ in the vertical profile).

Effective temperature for radiation:

\begin{tabular}{|c|c|c|}
\hline & Night & Day \\
\hline Sky & $250 \mathrm{~K}$ & N/A \\
\hline Enclosure interior surface & TA-1 K & TA+1 K \\
\hline
\end{tabular}

The effective sky temperature can vary by a few degrees following zenith angle variability but it is assumed that the structure senses an average value due to its thermal inertia.

Convective heat transfer coefficient:

\begin{tabular}{|c|c|}
\hline Night & Day \\
\hline $4 \mathrm{~W} / \mathrm{m}^{2} / \mathrm{K}$ & $2 \mathrm{~W} / \mathrm{m}^{2} / \mathrm{K}$ \\
\hline
\end{tabular}

The night time heat transfer coefficient can vary both in time and in space following telescope orientation variability and vent operation, but it is assumed that the structure senses an average value due to its thermal inertia. Values of up to 10 $\mathrm{W} / \mathrm{m}^{2} / \mathrm{K}$ have been estimated by CFD on certain parts of the structure, making the selected value a conservative one. 


\subsection{Material properties}

Table 1 summarizes the material properties used for the solid components of the model.

Table 1. Model material properties

\begin{tabular}{|c|c|c|c|c|}
\hline Property & $\begin{array}{c}\text { Duct } \\
(4 \mathrm{~mm} \mathrm{Al})\end{array}$ & $\begin{array}{c}\text { Frames } \\
(\text { steel })\end{array}$ & $\begin{array}{c}\text { Mirrors } \\
(\text { glass })\end{array}$ & $\begin{array}{c}\text { Lenses } \\
\text { (glass) }\end{array}$ \\
\hline Density $\mathrm{kg} / \mathrm{m}^{3}$ & 2700 & 8000 & 2500 & 2500 \\
\hline Conductivity W/m/K & 240 & 14 & 1.1 & 1.1 \\
\hline Specific heat J/kg/K & 900 & 500 & 850 & 850 \\
\hline Emissivity & $0.1^{*}$ & 0.8 & $0.8 / 0.02$ & $0.1(\tau=0.8)$ \\
\hline
\end{tabular}

For the top side of the duct above the hex ring level it is assumed that effort will be made to reduce the emissivity below 0.1 to avoid super-cooling. For the remaining duct surfaces it is assumed that effort will be made to minimize stray light reflections. That can potentially result in emissivity values over 0.8 , a conservative assumption. It should be mentioned that during preliminary simulations it was concluded that the $4 \mathrm{~mm}$ aluminum ductwork follows the ambient temperature better that the $2 \mathrm{~mm}$ steel option.

\subsection{Thermal boundary conditions}

The model is initialized at $\mathrm{T}=275.5 \mathrm{~K}$.

The only explicit heat source comes from the laser beams. Using a power loss of $0.5-1 \%$, depending on location and optical surface type, we estimated the power absorption of the optical components, which, expressed in power per unit volume, have the values of 1300 and $365 \mathrm{~W} / \mathrm{m}^{3}$ for the lenses and the mirrors respectively. As a worst case it is assumed that the lasers are operating the entire night.

\section{RESULTS}

Even though we are interested in night-time performance, diurnal simulations are necessary to ensure that the final result is independent of the initial conditions. The typical simulation lasts $36 \mathrm{~h}$ (night-day-night, each $12 \mathrm{~h}$ long). Only results after the first $24 \mathrm{~h}$ cycle are considered and reported. Such a long simulation cannot be performed at high sampling rates. The time-step used in the initial simulations was 300s. This is too coarse for beam jitter estimates but it is sufficient for temperature calculations. The resulting refractive index variations in time and space are more representative of beam wander. However, comparisons can still be made between the optical turbulence behavior of the two candidate paths.

In the following figures the results for the thermal environment inside the BTO are presented. Figure 6 shows the spatial distribution of temperature at the end of night, relative to the average interior air temperature, for the EJFA baseline path location. This location is particularly troublesome due to the proximity of the centering mirror support frame and the double relay lens arrays. It is worth noting that even though the spatial temperature gradients for a single mirror (or lens) are small, there can be differences of up to $0.5 \mathrm{~K}$ between mirrors. Figures 7 and 8 show the spatial distribution of temperature at the end of night, relative to the average interior air temperature, for two locations of the alternative path, TPA at the beginning of the path and HEXFA at the hex ring level. The temperature of the double lens arrays at the TPA alternative location is even higher than that of the baseline EJFA location. The velocities caused by buoyancy at the beginning of the path are small, resulting in less effective cooling by convection. The proximity of the array to the duct right above the enclosure in combination with the abrupt reduction in size restricts the flow, further reducing cooling and creating a pulsating flow effect, as can be seen in Figure 9. 


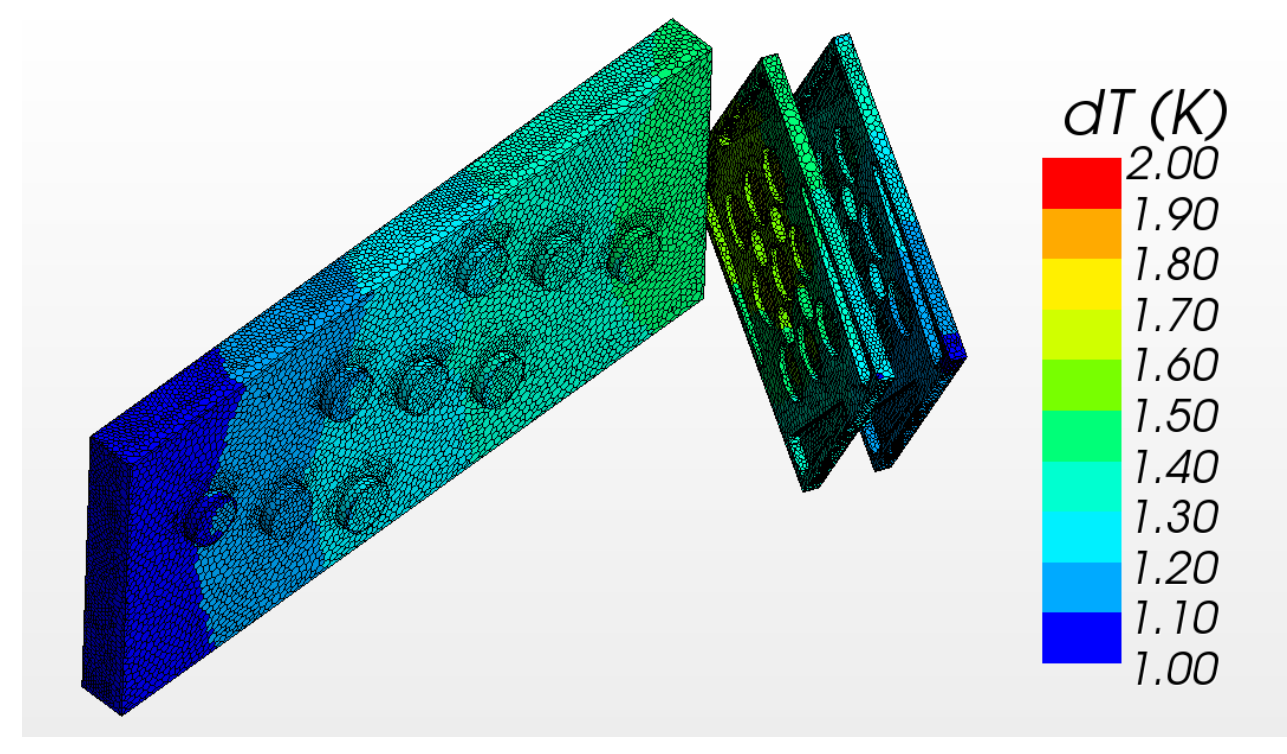

Figure 6. End of night temperature distribution relative to mean interior air at the baseline EJFA location. The grid surface resolution of $0.005 \mathrm{~m}$ is also shown.

The quantity of turbulent viscosity was chosen as it is directly tied to optical turbulence. Additional preliminary simulations at $1 \mathrm{~Hz}$ and $10 \mathrm{~Hz}$ verified that the effect has periods in excess of 10s, which the LGSF control loop should be able to effectively control. However, design changes have already been made. All distances between mirror and lens frames as well as frames and enclosure walls have been increased. For the alternative TPA location in particular the transition from enclosure diameter to duct diameter is now achieved through a conic duct.

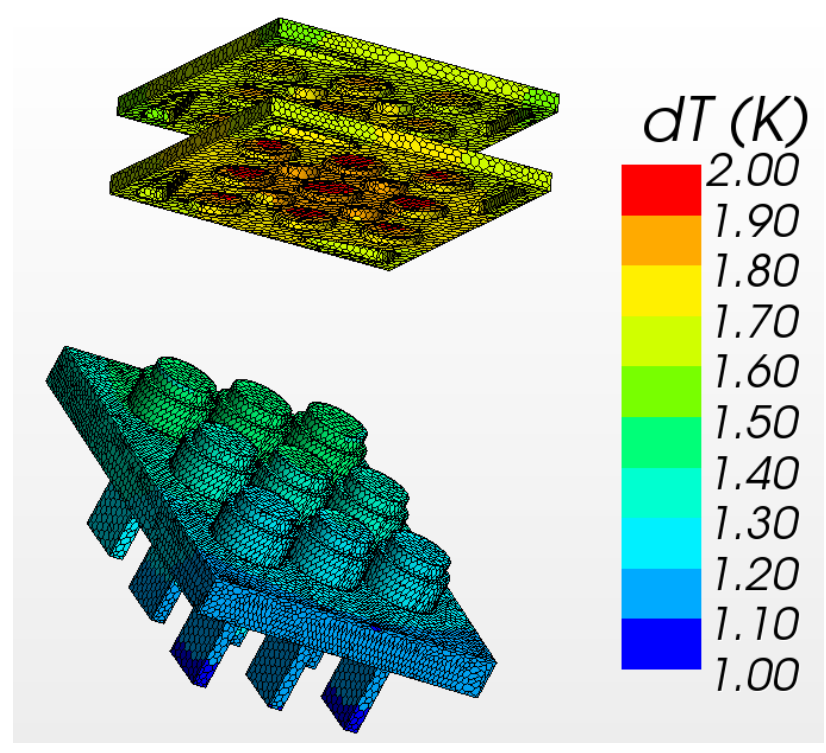

Figure 7. End of night temperature distribution relative to mean interior air at the alternative TPA location (with grid resolution) 


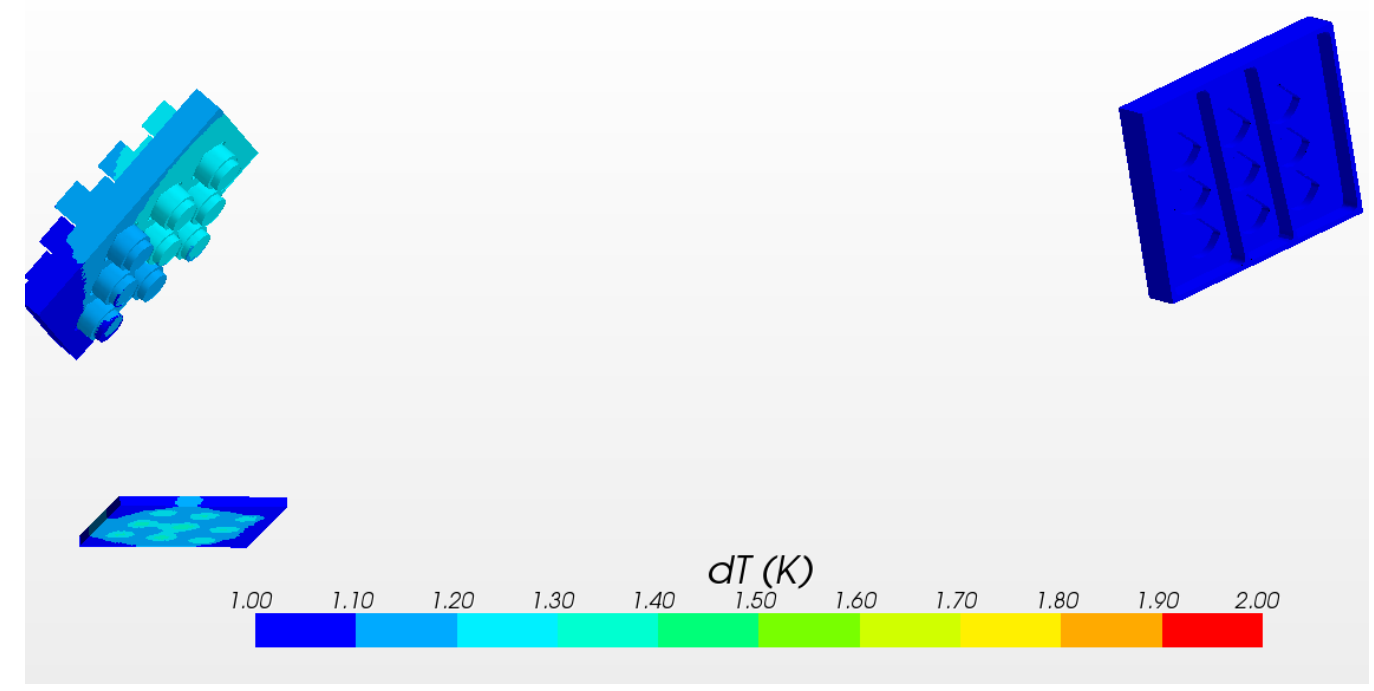

Figure 8. End of night temperature distribution relative to mean interior air at the alternative HEXFA locations

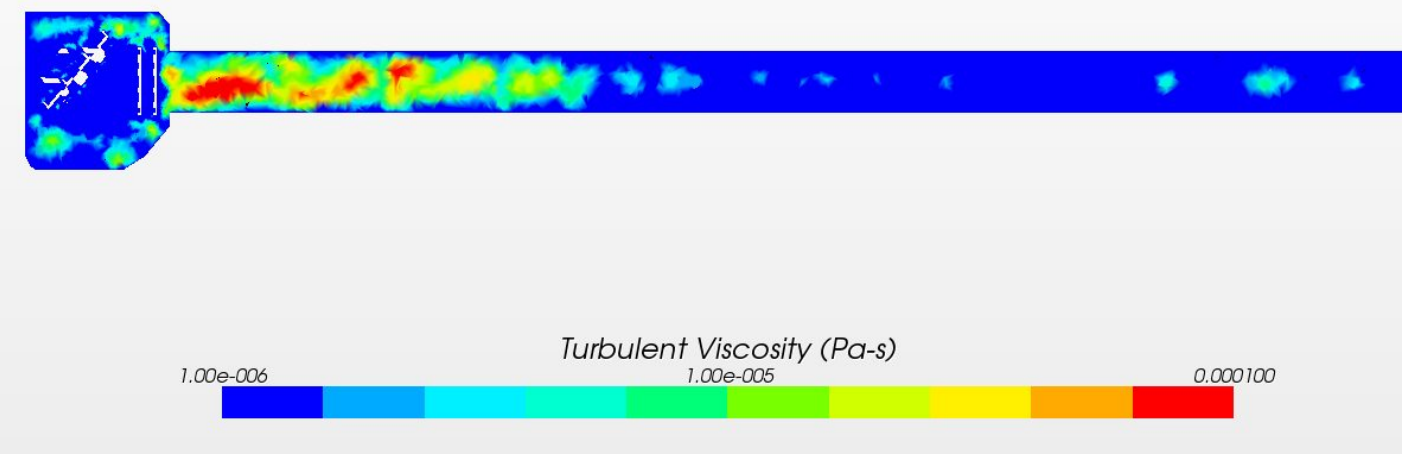

Figure 9. End of night turbulent viscosity at the alternative TPA location showing pulsating "chimney" effect. This location is responsible for most of the refractive index variance along the optical path.

The overall night-time temperature behavior of the different regions is presented in Figure 10. The behavior is almost identical for both paths. The interior duct air temperature in effect acquires by means of shear convection through a large surface the duct exterior wall temperature (green and blue curves). The latter, as explained, depends on the emissivity and prevailing convective heat transfer coefficient, for which conservative values have been used. It is expected that the currently shown $\sim 1 \mathrm{~K}$ difference from ambient (black curve) will be reduced.

Finally, Figure 11 provides a first comparison between the two candidate paths' optical performance. The spatial refractive index variance of the alternative path (blue curve) is $70 \%$ or less than that of the baseline path (red curve) throughout the night. Moreover, its length is shorter than the baseline length by $9 \%$. Beam jitter is proportional to both of these quantities. 


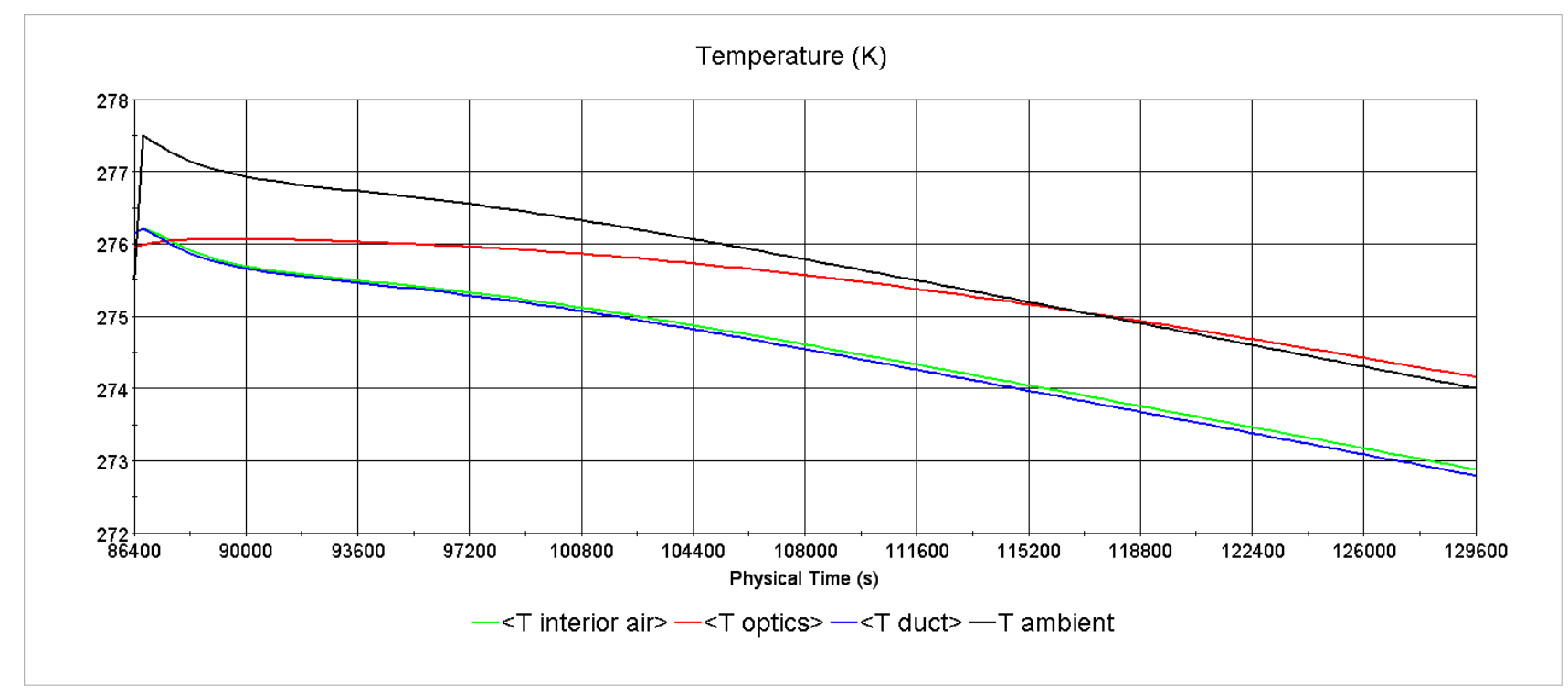

Figure 10. Night-time temporal behavior of temperature (baseline OP shown, alternative OP almost identical)

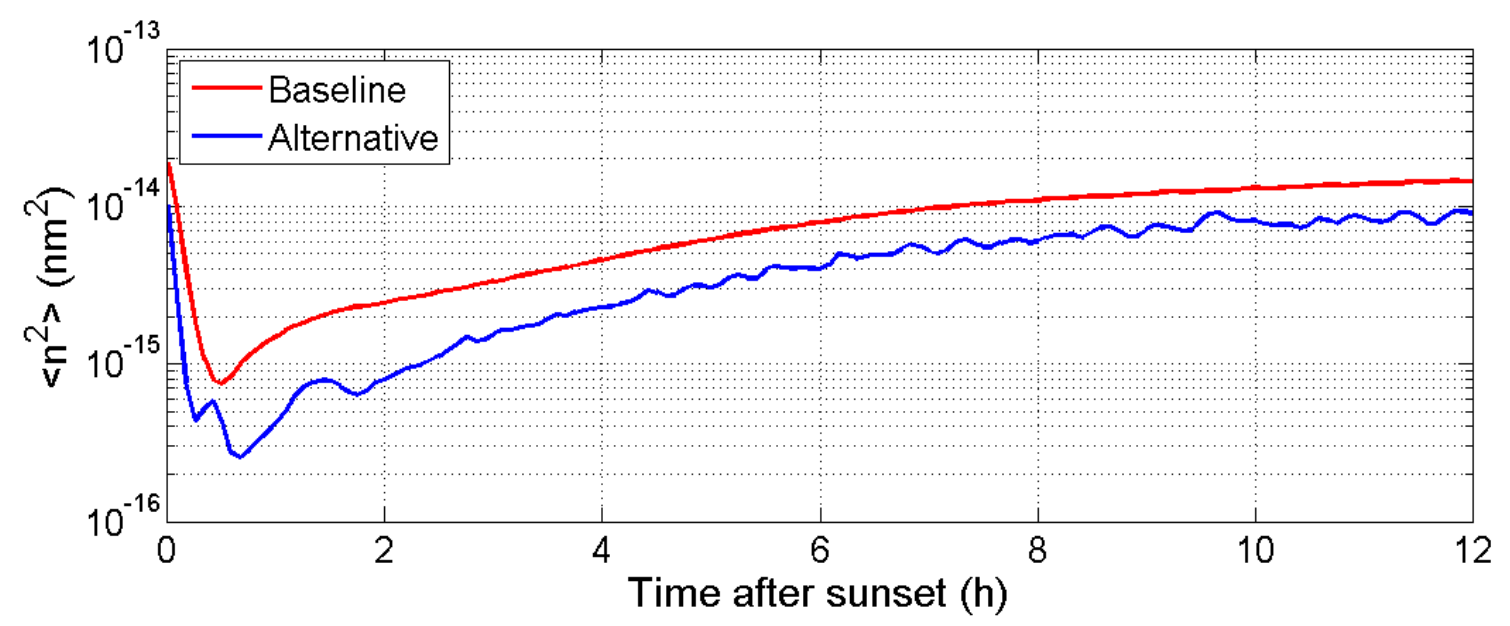

Figure 11. Night-time temporal behavior of the refractive index spatial variance $<\mathrm{n}^{2}>$, along the center beam

\section{CONCLUSIONS}

A Computational Solid Fluid Dynamics (CSFD) model of the TMT LGSF ducts has been developed and applied to both candidate optical paths.

The spatially averaged temperature temporal behavior of optics is similar for both paths.

The main causes for the optical turbulence appear to be locations with double relay lens arrays or array support frames close to each other or the ducts and fold enclosure walls. Design guidance has been provided and mitigation steps have already been implemented.

The new proposed path (alternative OP) appears overall better by optical turbulence considerations. As a next step, higher sampling rate simulations will be performed for a short night time period, when worst performance is expected (end of night) in order to quantify beam jitter for requirement compliance estimates (see also [8] for a somewhat relevant study). 


\section{ACKNOWLEDGMENT}

The TMT Project gratefully acknowledges the support of the TMT collaborating institutions. They are the Association of Canadian Universities for Research in Astronomy (ACURA), the California Institute of Technology, the University of California, the National Astronomical Observatory of Japan, the National Astronomical Observatories of China and their consortium partners, and the Department of Science and Technology of India and their supported institutes. This work was supported as well by the Gordon and Betty Moore Foundation, the Canada Foundation for Innovation, the Ontario Ministry of Research and Innovation, the National Research Council of Canada, the Natural Sciences and Engineering Research Council of Canada, the British Columbia Knowledge Development Fund, the Association of Universities for Research in Astronomy (AURA), the U.S. National Science Foundation and the National Institutes of Natural Sciences of Japan.

\section{REFERENCES}

[1] Sanders, G. H., "Thirty Meter Telescope project update," SPIE 9145(48), (2014).

[2] Boyer, C., et al, "Adaptive Optics Program at TMT," SPIE 9148(32), (2014).

[3] Vogiatzis, K., Sadjadpour, A., Roberts, S., "TMT Telescope Structure thermal model," SPIE 9150(72), (2014).

[4] Vogiatzis, K., "Transient aero-thermal simulations for TMT," SPIE 9150(24), (2014).

[5] Vogiatzis, K., Angeli, G. Z., "Monte Carlo Simulation Framework for TMT," SPIE 7017(29), (2008).

[6] Vogiatzis, K., "Aero-thermal modeling framework for TMT," SPIE 8336(10), (2011).

[7] Thompson, H., Vogiatzis, K., "Heat balance and thermal management of the TMT Observatory," SPIE 9150(83), (2014).

[8] Vogiatzis, K., Otarola, A., Skidmore, W., Travouillon, T., Angeli, G. Z., "Thermal seeing modeling validation through observatory measurements," SPIE 8449(1), (2012). 